\title{
Cengentán
}

\section{¿Qué publica Brasil en Biblioteconomía y Documentación? \\ Estudio de la producción internacional y análisis de Clúster Bibliográficos (Web of Science 2000-2014)}

\author{
Daniela De Filippo \\ Doctora; Universidad Carlos III de Madrid, Madrid, Espanha; \\ dfilippo@bib.uc3m.es
}

\begin{abstract}
Resumen: Se analizan las publicaciones de Brasil en Library and Information Science de Web of Science. El objetivo es conocer las características de la producción, analizar el perfil de actividad del país y detectar el corpus documental usado como marco conceptual. La metodología consta de dos etapas: en la primera se recogió la producción y se obtuvieron los principales indicadores bibliométricos de actividad, especialización y colaboración. En la segunda, se utilizó el método de bibliographic coupling para detectar "comunidades bibliográficas" con referencias comunes. Los centros brasileños publicaron 910 documentos entre 2000 y 2014, especialmente en portugués, siendo las revistas editadas en el país las principales vías de difusión. Se detectaron clusters bibliográficos relacionados con estudios sobre Open Access, recuperación de información, estudios métricos, organización del conocimiento y cienciometría. Esta última comunidad es la más internacional, tanto por las revistas de consumo como de difusión.
\end{abstract}

Palabras clave: Producción científica. Clusters bibliográficos. Información y documentación. Brasil.

\section{Introducción}

El campo de la Biblioteconomía y Documentación puede presentar características y dinámicas diferentes según el contexto en el que se desarrolle. Desde su institucionalización en los años 1960, con las primeras escuelas de biblioteconomía, las actividades de investigación en esta área han ido evolucionando de manera diferente en cada país. En algunos se han vinculado académicamente a las Ciencias de la Información, en otros han formado parte de facultades de Documentación o de Comunicación. A pesar de estas diferencias a nivel local, a la hora de difundir internacionalmente los resultados de la investigación los profesionales del campo se orientan generalmente a revistas incluidas en la disciplina 


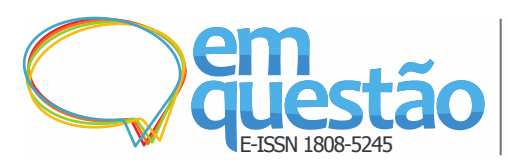

¿Qué publica Brasil en Biblioteconomía y documen-

tación? Estudio de la producción internacional y

análisis de Clúster Bibliográficos (Web of Science 2000-

2014)

Daniela De Filippo

Information Science \& Library Science (LIS). Es así que una de las principales fuentes de información para analizar la producción internacional en este campo es la Web of Science (WoS) que cuenta con publicaciones en esta categoría desde 1945. Aunque la producción internacional se concentra principalmente en los países anglosajones, las publicaciones de Brasil en este campo van ganando posiciones. Así, en la última década, la producción del país en LIS lo ubica en la décima posición del mundo, mientras que por su producción total en WoS, según los Essential Science Indicators, Brasil se encuentra en el puesto 14 (THOMSON REUTERS, 2015a, 2015b).

Hasta el momento diferentes estudios han analizado las principales características de las publicaciones internacionales en Information Science \& $\mathrm{Li}$ brary Science desde una perspectiva bibliométrica (LEVITT; THELWALL, 2009; NIXON, 2014), algunos de los cuales se han centrado en el caso específico de Brasil. En este sentido, el estudio de la producción científica ha sido abordado desde diferentes perspectivas. Algunos autores se focalizaron en la importancia de las revistas de Biblioteconomía brasileña como fuente para la investigación (FORESTI, 1990). Trabajos más recientes se han centrado en la evaluación de la producción científica de las revistas de Ciencias de la Información (OLIVEIRA; MORAES, 2008), otros han analizado los perfiles de actividad en el campo a través del estudio de los curricula de los investigadores (SOUZA; FERREIRA, 2013) y otros han estudiado revistas específicas de esta disciplina (PINTO; SANTOS; BAHIA, 2009; ANDRÉ et al., 2014).

Utilizando técnicas bibliométricas, en este trabajo nos proponemos dar un paso más e indagar en las relaciones intertextuales utilizando técnicas de $b i$ bliographic coupling para identificar las llamadas "comunidades bibliográficas" que constituyen el corpus documental producido por instituciones de Brasil en LIS. Así, a través de la detección de textos que comparten referencias comunes, se pretende conocer mejor la estructura de la investigación en este contexto y analizar su dinámica a lo largo del tiempo.

A través de este estudio se intentará dar respuesta a los siguientes interrogantes descriptivos y analíticos: 
Daniela De Filippo

a) ¿Cómo ha sido la evolución de la producción LIS brasileña en los últimos 15 años?;

b) ¿Esta producción presenta características estructurales (idioma, tipo documental, revistas de publicación, etc.) similares a las del resto del mundo?;

c) ¿Cuáles son los principales referentes teóricos de las publicaciones LIS de Brasil?;

d) ¿Ha evolucionado el consumo de revistas internacionales del campo?. Asimismo, con esta metodología es posible identificar los principales actores institucionales y explorar las redes temáticas que conforman las publicaciones brasileñas en este campo.

\section{Fuentes y metodología}

Se han utilizado como fuente de información las publicaciones incluidas en la Web of Science (WoS). A pesar de las conocidas limitaciones de esta fuente - en cuanto a sus sesgos temáticos, idiomáticos y geográficos - se ha decidido utilizarla porque ofrece información desagregada por disciplina y permite seleccionar de manera bastante acotada la producción en Library \& Information Science. Asimismo, incluye la adscripción institucional de todos los autores de los documentos, lo que resulta fundamental para el estudio de la colaboración científica. Este estudio se desarrolló principalmente en dos fases.

(1) En primer lugar, se identificó la producción científica de Brasil en Web of Science utilizando la estrategia $(\mathrm{CU}=$ Brazil AND WC=Information Science \& Library Science). A continuación se construyó una base de datos relacional en MySQL con las publicaciones recogidas de la WoS. Tras depurar y normalizar la información, se obtuvieron los siguientes indicadores bibliométricos:

a) de actividad - número de documentos por año, idioma de publicación, tipología documental, revistas;

b) de especialización - distribución disciplinar, grado de solapamiento con otras disciplinas;

c) de colaboración - número y porcentaje de documentos en colaboración internacional, principales países colaboradores. 


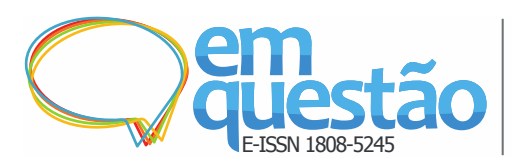

¿Qué publica Brasil en Biblioteconomía y documen-

tación? Estudio de la producción internacional y

análisis de Clúster Bibliográficos (Web of Science 2000-

2014)

Daniela De Filippo

Estos mismos indicadores han sido obtenidos para toda la producción mundial en LIS para poder comparar el perfil de actividad brasileña con el del mundo. El período analizado ha sido 2000-2014.

(2) La segunda fase se centra en el estudio del área a partir del análisis de los clusters que conforman los textos con referencias compartidas. Se utiliza la herramienta BiblioToolbox desarrollada por Grauwin y Jensen (GRAUWIN; JENSEN, 2011; GRAUWIN et al., 2012). A partir del concepto bibliométrico de bibliographic coupling (KESSLER, 1963) esta herramienta transforma la información bibliográfica obtenida de Web of Science en "mapas de la ciencia”. Para ello extrae conjuntos coherentes de información (autores, instituciones, palabras clave, áreas temáticas, revistas de publicación, etc.) de las publicaciones que conforman los datos de análisis a partir de las publicaciones que comparten referencias. Los grafos que se obtienen como resultado pueden ser fácilmente representados con herramientas del análisis de redes sociales (ARS/SNA), como Gephi. Esta metodología, diseñada en lenguaje de programación Python (GRAUWIN, c2012) permite mejorar las limitaciones de interpretación que conllevan los habituales mapas simples de co-ocurrencias (co-autoría; co-palabras o co-citación) presentando en un único grafo denominado 'mapa heterogéneo' todas las co-ocurrencias simultáneamente.

\section{Resultados}

Los resultados se dividen en las fases 1 y 2 .

\subsection{Fase 1}

La producción mundial recogida en Library \& Information Science entre los años 2000 y 2014 consta de 149.585 publicaciones. Un 58\% corresponde a Estados Unidos seguido de Inglaterra y Canadá con un $4 \%$ y $3 \%$ respectivamente. Brasil, con 910 documentos, aporta el 0,61\% del mundo y se ubica en el puesto 11 (Figura 1). 


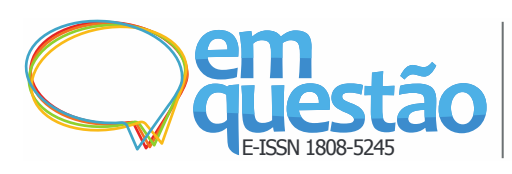

¿Qué publica Brasil en Biblioteconomía y documentación? Estudio de la producción internacional y análisis de Clúster Bibliográficos (Web of Science 20002014)

Daniela De Filippo

Figura 1 - Distribución de la producción en Library \& Information Science por país.

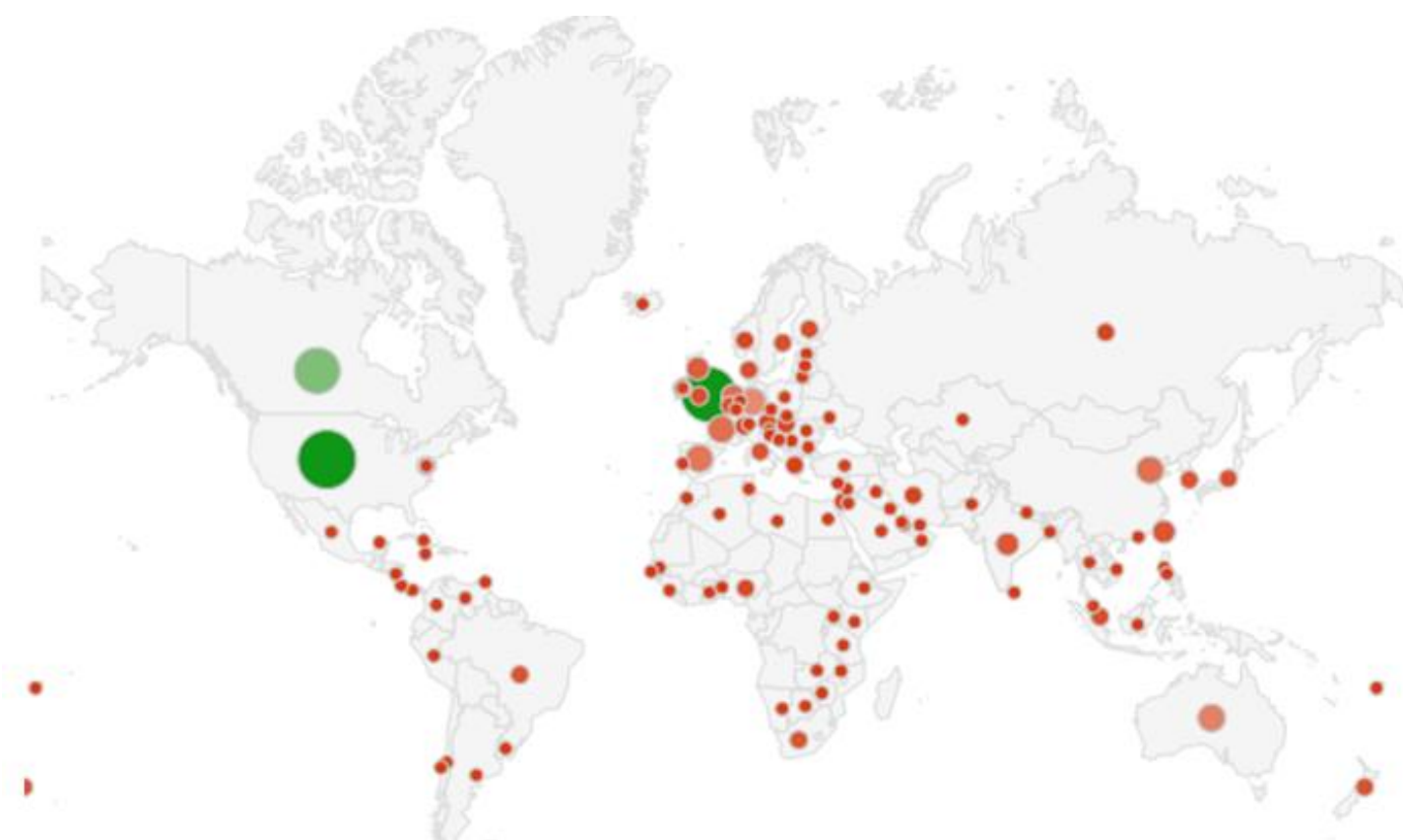

Fuente: Elaboración propia a partir de datos obtenidos de Web of Science

Al considerar la evolución anual del número de documentos de Brasil, se observa que un $85 \%$ de ellos se publican a partir del año 2008. Si bien la aportación del país a la producción mundial puede resultar escasa en términos absolutos, los porcentajes de publicaciones se han ido incrementando desde el 0,002\% en el año 2000 al 0,010\% en 2014 (Tabla 1).

Tabla 1 - Evolución del número de documentos LIS de Brasil y del mundo por año.

(WoS 2000-2014)

\begin{tabular}{cccccc}
\hline \multirow{2}{*}{ Año } & \multicolumn{2}{c}{ BRASIL } & \multicolumn{2}{c}{ MUNDO } & \multirow{2}{*}{ Brasil/mundo } \\
\cline { 2 - 4 } & N docs & \% Total & N docs & \% Total & \\
\hline 2000 & 21 & 2,31 & 10989 & 7,35 & 0,002 \\
2001 & 17 & 1,87 & 10829 & 7,24 & 0,002 \\
2002 & 4 & 0,44 & 10285 & 6,88 & 0,000 \\
2003 & 13 & 1,43 & 10755 & 7,19 & 0,001 \\
2004 & 10 & 1,10 & 9991 & 6,68 & 0,001 \\
2005 & 14 & 1,54 & 10212 & 6,83 & 0,001 \\
2006 & 33 & 3,63 & 10145 & 6,78 & 0,003 \\
2007 & 23 & 2,53 & 10074 & 6,73 & 0,002 \\
2008 & 114 & 12,53 & 9876 & 6,60 & 0,012 \\
2009 & 117 & 12,86 & 8844 & 5,91 & 0,013 \\
\hline
\end{tabular}




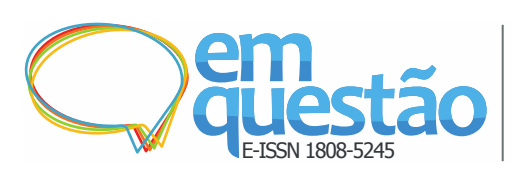

¿Qué publica Brasil en Biblioteconomía y documentación? Estudio de la producción internacional y análisis de Clúster Bibliográficos (Web of Science 20002014)

Daniela De Filippo

\begin{tabular}{cccccc}
\hline \multirow{2}{*}{ Año } & \multicolumn{2}{c}{ BRASIL } & \multicolumn{2}{c}{ MUNDO } & \multirow{2}{*}{ Brasil/mundo } \\
\cline { 2 - 5 } & N docs & \% Total & N docs & \% Total & \\
\hline 2010 & 112 & 12,31 & 9594 & 6,41 & 0,012 \\
2011 & 111 & 12,20 & 9628 & 6,44 & 0,012 \\
2012 & 133 & 14,62 & 9076 & 6,07 & 0,015 \\
2013 & 89 & 9,78 & 9558 & 6,39 & 0,009 \\
2014 & 99 & 10,88 & 9729 & 6,50 & 0,010 \\
\hline Total & 910 & 100,00 & 149585 & 100,00 & 0,006 \\
\hline
\end{tabular}

Fuente: Elaboración propia a partir de datos obtenidos de Web of Science

La producción científica brasileña en LIS se ha difundido en 64 revistas siendo las primeras: Informação \& Sociedade: Estudos, Perspectivas em Ciência da Informação y Transinformação - las tres brasileñas - que en conjunto concentran un $62 \%$ de la producción hecha en el país en este campo. En el mundo, en los 15 años analizados, se ha publicado en 117 revistas entre las que destacan: Library Journal (53\%), Scientist (5\%) y Journal of the American Medical Informatics Association (1,8\%). En la tabla 2 se pueden observar estos valores y detectar que los documentos de Brasil tienen porcentajes de publicación en revistas editadas en el país muchos más elevados que los del resto de mundo.

Tabla 2 - Revistas de publicación LIS de Brasil y del mundo (WoS 2000-2014).

\begin{tabular}{rrcc}
\multicolumn{1}{c}{ Revista } & N doc BR & \% BR & \% Mundo \\
\hline Informação \& Sociedade: Estudos & 249 & 27,36 & 0,20 \\
Perspectivas em Ciência da Informação & 193 & 21,21 & 0,21 \\
Transinformação & 125 & 13,74 & 0,10 \\
Scientometrics & 62 & 6,81 & 17,13 \\
Scientist & 26 & 2,86 & 5,22 \\
Knowledge Organization & 25 & 2,75 & 0,31 \\
\hline Information Processing Management & 18 & 1,98 & 0,78 \\
\hline cournal of the American Medical Informatics Asso- & 24 & 2,64 & 18,48 \\
Science and Technology & 17 & 1,87 & 17,11 \\
\hline Journal of the American Society for Information & 11 & 1,21 & 0,30 \\
\hline Social Science Information sur les Sciences Sociales & 10 & 1,10 & 0,25 \\
Journal of Knowledge Management & 10 & 0,99 & 0,77 \\
\hline Information Research an International Electronic & 9 & & \\
\hline Jnternational Journal of Geographical Information & 9 & 0,99 & 0,82 \\
Science & & & 0,66 \\
\hline International Journal of Information Management & 8 & 0,88 & \\
\hline
\end{tabular}


Daniela De Filippo

\begin{tabular}{|c|c|c|c|}
\hline Revista & $\mathbf{N} \operatorname{doc} \mathbf{B R}$ & $\% \mathbf{B R}$ & \% Mundo \\
\hline Investigación Bibliotecológica & 8 & 0,88 & 0,17 \\
\hline Journal of Informetrics & 8 & 0,88 & 0,34 \\
\hline Profesional de la Información & 7 & 0,77 & 0,49 \\
\hline Knowledge Management Research Practice & 6 & 0,66 & 0,18 \\
\hline Information Development & 5 & 0,55 & 0,16 \\
\hline Journal of Global Information Management & 5 & 0,55 & 0,12 \\
\hline $\begin{array}{r}\text { Journal of the aAssociation for Information Science } \\
\text { and Technology }\end{array}$ & 5 & 0,55 & 0,14 \\
\hline Lecture Notes in Computer Science & 5 & 0,55 & 0,18 \\
\hline $\begin{array}{r}\text { Research and Advanced Technology for Digital } \\
\text { Libraries }\end{array}$ & 5 & 0,55 & 0,09 \\
\hline Research Evaluation & 5 & 0,55 & 0,29 \\
\hline Government Information Quarterly & 4 & 0,44 & 0,64 \\
\hline Proceedings of the ASIST Annual Meeting & 4 & 0,44 & 0,24 \\
\hline $\begin{array}{l}\text { Restaurator International Journal for the Preserva- } \\
\text { tion of Library and Archival Material }\end{array}$ & 4 & 0,44 & 0,19 \\
\hline $\begin{array}{r}\text { ASIST 2003: Proceedings of the } 66^{\text {th }} \text { ASIST Annual } \\
\text { Meeting, vol. } 40\end{array}$ & 3 & 0,33 & 0,80 \\
\hline $\begin{array}{r}\text { Canadian Journal of Information and Library } \\
\text { Science/ Revue Canadienne des Sciences de } \\
\text { l'Iinformation et de Bibliothéconomie }\end{array}$ & 3 & 0,33 & 0,47 \\
\hline Health Information and Libraries Journal & 3 & 0,33 & 0,35 \\
\hline Information Society & 3 & 0,33 & 0,44 \\
\hline Journal of Health Communication & 3 & 0,33 & 0,84 \\
\hline Journal of Information Technology & 3 & 0,33 & 0,29 \\
\hline Journal of Strategic Information Systems & 3 & 0,33 & 0,22 \\
\hline Telecommunications Policy & 3 & 0,33 & 0,69 \\
\hline Aslib Proceedings & 2 & 0,22 & 0,37 \\
\hline Information Technology for Development & 2 & 0,22 & 0,09 \\
\hline $\begin{array}{r}\text { International Journal of Computer sSupported } \\
\text { Collaborative Learning }\end{array}$ & 2 & 0,22 & 0,14 \\
\hline Journal of Documentation & 2 & 0,22 & 0,73 \\
\hline Journal of the Medical Library Association & 2 & 0,22 & 0,79 \\
\hline MIS Quarterly Executive & 2 & 0,22 & 0,09 \\
\hline Revista española de Documentación Científica & 2 & 0,22 & 0,17 \\
\hline Social Science Computer Review & 2 & 0,22 & 0,46 \\
\hline Telematics and Informatics & 2 & 0,22 & 0,11 \\
\hline $\begin{array}{r}\text { ASIST 2002: Proceedings of the } 65^{\text {th }} \text { ASIST Annual } \\
\text { Meeting, vol. } 39\end{array}$ & 1 & 0,11 & 0,08 \\
\hline Electronic Library & 1 & 0,11 & 0,99 \\
\hline Információs Társadalom & 1 & 0,11 & 0,17 \\
\hline Information and Organization & 1 & 0,11 & 0,07 \\
\hline Information Culture & 1 & 0,11 & 0,04 \\
\hline Information Management & 1 & 0,11 & 0,58 \\
\hline Information Technology and Libraries & 1 & 0,11 & 0,32 \\
\hline Information Technology Management & 1 & 0,11 & 0,12 \\
\hline
\end{tabular}




\begin{tabular}{|c|ccc}
\hline Revista & $\begin{array}{l}\text { iQué publica Brasil en Biblioteconomía y documen- } \\
\text { tación? Estudio de la producción internacional y } \\
\text { análisis de Clúster Bibliográficos } \\
\mathbf{2 0 1 4} \text { (Web of Science 2000- } \\
\text { Daniela De Filippo }\end{array}$ & & \\
\hline Information Technology People & 1 & 0,11 & 0,08 \\
\hline International Information Library Review & 1 & 0,11 & 0,03 \\
\hline Journal of Information Science & 1 & 0,11 & 0,52 \\
\hline Learned Publishing & 1 & 0,11 & 0,56 \\
\hline Library Collections Acquisitions Technical Services & 1 & 0,11 & 0,41 \\
\hline Library Journal & 1 & 0,11 & 53,42 \\
\hline Online Information Review & 1 & 0,11 & 0,98 \\
\hline Publishing Research Quarterly & 1 & 0,11 & 0,13 \\
\hline Serials Review & 1 & 0,11 & 0,34 \\
\hline
\end{tabular}

Fuente: Elaboración propia a partir de datos obtenidos de Web of Science

En cuanto al idioma de publicación, más de la mitad de las publicaciones brasileñas son en portugués y un tercio en inglés, mientras que, en el resto del mundo, el portugués representa sólo el $0,49 \%$ de las publicaciones siendo el inglés ampliamente dominante (Tabla 3 ).

Tabla 3 - Idioma de publicación de los documentos (WoS 2000-2014).

\begin{tabular}{rcccc}
\hline \multirow{2}{*}{ Idioma } & \multicolumn{2}{c}{ Brasil } & \multicolumn{2}{c}{ Mundo } \\
\cline { 2 - 5 } & N docs & $\%$ & N docs & $\%$ \\
\hline Portugués & 538 & 59,12 & 727 & 0,49 \\
Inglés & 334 & 36,79 & 145.993 & 97,60 \\
Español & 32 & 3,52 & 1259 & 0,84 \\
Francés & 05 & 0,55 & 142 & 0,09 \\
Alemán & 00 & 00 & 1095 & 0,73 \\
Otros & 01 & 0,11 & 376 & 0,25 \\
\hline
\end{tabular}

Fuente: Elaboración propia a partir de datos obtenidos de Web of Science

En relación a la especialización temática, si bien la totalidad de la producción corresponde a la categoría Library \& Information Science, la multiclasificación de revistas produce un solapamiento entre wos categories. En este sentido, la producción se ha recogido, además de en LIS, en otras 16 categorías. Entre ellas destacan: Computer Science Information System y Computer Science 
Daniela De Filippo

Interdisciplinary Applications (con un $21 \%$ de los documentos), seguidas de Management, Multidisciplinary Science y Medical Informatics. En la producción del mundo se han detectado 24 categorías (además de LIS) coincidiendo las principales con las que predominan en Brasil (figura 2).

Figura 2 - Disciplinas de muticlasificación de la producción en Library \& Information Science

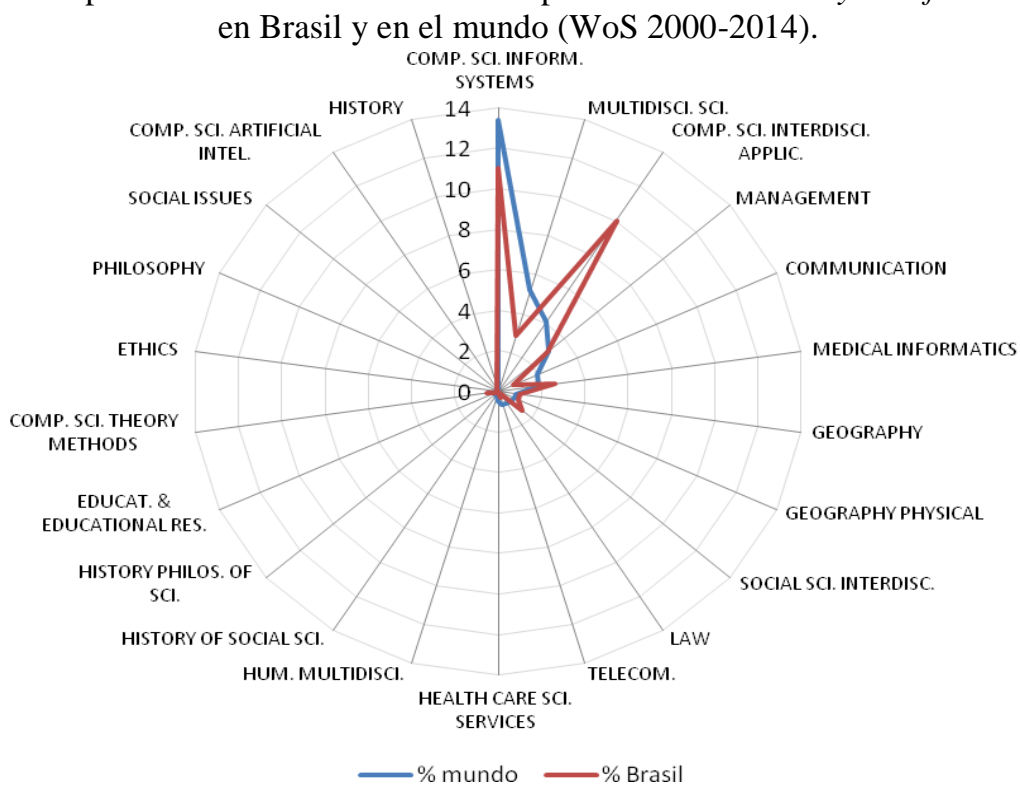

Fuente: Elaboración propia a partir de datos obtenidos de Web of Science

Al analizar la colaboración internacional se aprecia que un $18 \%$ de la producción brasileña ha sido elaborada junto a otros países. Si bien se han detectado publicaciones junto a 34 países, los principales socios han sido Estados Unidos y España, participando cada uno en un 5\% de los documentos de Brasil. Le siguen, aunque con menor volumen de documentos conjuntos: Canadá, Inglaterra y Francia (Figura 3). 
Daniela De Filippo

Figura 3 - Principales países colaboradores de Brasil (WoS 2000-2014).

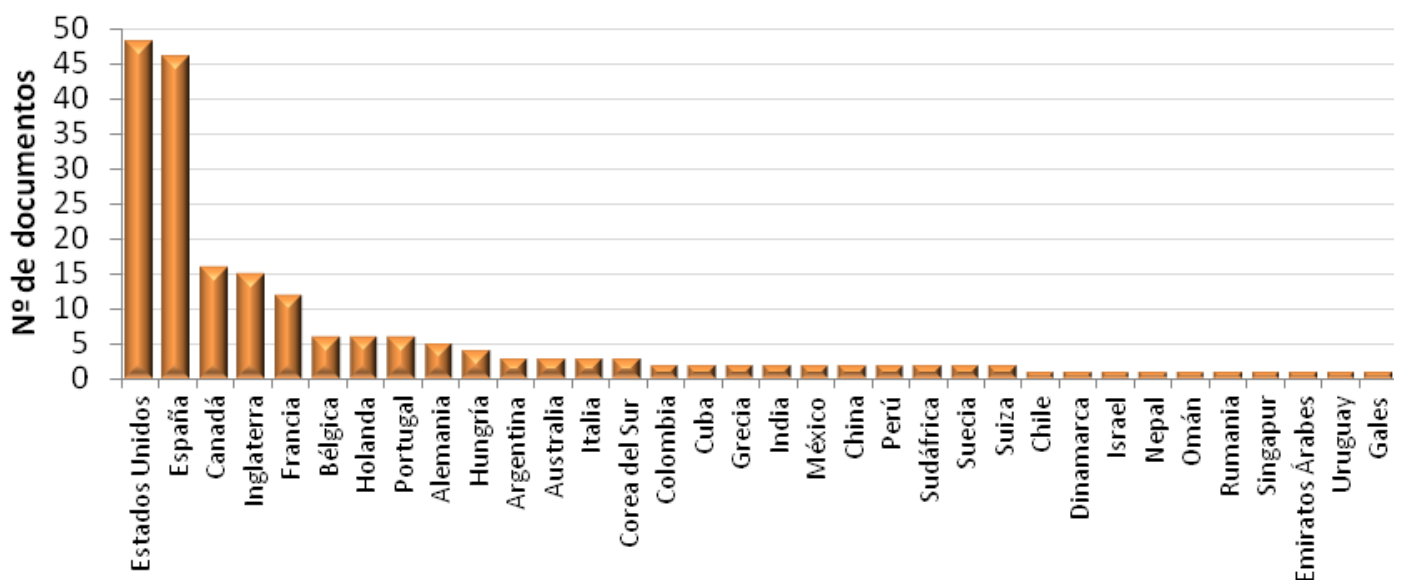

Fuente: Elaboración propia a partir de datos obtenidos de Web of Science

A nivel de instituciones, en Brasil son las universidades las principales productoras de publicaciones. Destacan especialmente la Universidad Federal de Minas Gerais y la Universidad de São Paulo (Tabla 4). A nivel internacional son también las universidades y las bibliotecas públicas las principales instituciones que publican en LIS. En las primeras posiciones se ubican instituciones de Estados Unidos como los sistemas universitarios de New York, California y Pennsylvania.

Tabla 4 - Principales instituciones productoras de Brasil en LIS ( $>5$ documentos). (WoS 2000-2014).

\begin{tabular}{rcc}
\hline Institución & N doc & \% \\
\hline Universidade Federal de Minas Gerais (UFMG) & 151 & 16,59 \\
Universidade de São Paulo (USP) & 128 & 14,07 \\
Universidade federal do rio de janeiro (UFRJ) & 83 & 9,12 \\
Universidade Federal de Santa Catarina (UFSC) & 72 & 7,91 \\
Universidade Federal da Paraíba (UFPB) & 71 & 7,80 \\
Universidade de Brasília (UnB) & 61 & 6,70 \\
Universidade Estadual Paulista (UNESP) & 57 & 6,26 \\
Universidade Estadual de Campinas (UNICAMP) & 39 & 4,29 \\
Universidade Federal do Rio Grande do Sul (UFRGS) & 38 & 4,18 \\
Universidade Federal do Paraná (UFPR) & 29 & 3,19 \\
Universidade Federal da Bahia (UFBA) & 24 & 2,64 \\
Universidade Federal Fluminense (UFF) & 22 & 2,42 \\
\hline
\end{tabular}


Daniela De Filippo

\begin{tabular}{|c|c|c|}
\hline Institución & N doc & $\%$ \\
\hline Universidade do Estado do Rio de Janeiro (UERJ) & 21 & 2,31 \\
\hline Universidade Federal de São Carlos (UFSCar) & 20 & 2,20 \\
\hline Universidade Federal de Santa Maria (UFSM) & 18 & 1,98 \\
\hline Pontifícia Universidade Católica de Campinas (PUC- & 17 & 1,87 \\
\hline \multicolumn{3}{|l|}{ Campinas) } \\
\hline Fundação Oswaldo Cruz (Fiocruz) & 15 & 1,65 \\
\hline Fundação Getúlio Vargas (FGV) & 14 & 1,54 \\
\hline Universidade Estadual de Londrina (UEL) & 14 & 1,54 \\
\hline Universidade Federal de Pernambuco (UFP) & 14 & 1,54 \\
\hline Pontifícia Universidade Católica de Minas Gerais (PUC-MG) & 9 & 0,99 \\
\hline Universidade Federal do Amazonas (UFAM) & 9 & 0,99 \\
\hline Universidade Federal de São Paulo (UNIFESP) & 9 & 0,99 \\
\hline Universidade Federal do Ceará (UFC) & 9 & 0,99 \\
\hline Universidade Federal de Goiás (UFG) & 7 & 0,77 \\
\hline $\begin{array}{r}\text { Conselho Nacional de Desenvolvimento Científico e Tecnológi- } \\
\text { co } \\
(\mathrm{CNPq})\end{array}$ & 6 & 0,66 \\
\hline $\begin{array}{r}\text { Instituto Nacional de Pesquisas Espaciais } \\
\text { (INPE) }\end{array}$ & 6 & 0,66 \\
\hline Universidade Católica de Brasília (UCB) & 6 & 0,66 \\
\hline Universidade do Estado de Santa Catarina (UFSC) & 6 & 0,66 \\
\hline Universidade do Vale do Rio dos Sinos (UNISINOS) & 6 & 0,66 \\
\hline
\end{tabular}

Fuente: Elaboración propia a partir de datos obtenidos de Web of Science

\subsection{Fase 2}

La aplicación de técnicas de clustering realizadas en la segunda fase permitió obtener 10 comunidades bibliográficas relevantes en Brasil. Son consideradas relevantes las comunidades con más de 10 documentos que comparten un mínimo umbral de referencias comunes. A continuación se muestran las tablas obtenidas con el programa BiblioTools y que permiten observar las características de cada comunidad. Sólo se presentan las tres primeras ya que concentran más de 100 documentos cada una y muestran un perfil bastante diferenciado.

En el conjunto bibliográfico de Brasil la comunidad que agrupa un mayor número de publicaciones se muestra en la tabla 5. Como se puede apreciar, incluye 123 artículos vinculados con la temática "innovación”. Se observa que entre las principales palabras clave se encuentran: innovation, management, per- 


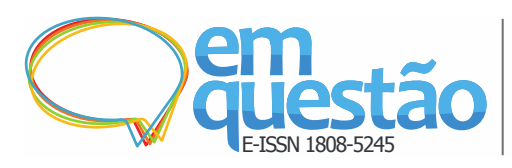

¿Qué publica Brasil en Biblioteconomía y documen-

tación? Estudio de la producción internacional y

análisis de Clúster Bibliográficos (Web of Science 2000-

2014)

Daniela De Filippo

formance, que dan una idea de la orientación de las publicaciones de este conjunto documental. Esto se deduce de las primeras columnas de la tabla donde se presentan las keywords más frecuentes. Se muestra la frecuencia de cada palabra clave (f\%) y la significación que representa la especificidad de un término en esa comunidad con respecto al total de documentos analizados. En este caso, innovation es el término más frecuente $(\mathrm{f}=6,59 \%)$, con una significación de 1,02. Es decir que aparece en esta comunidad con una frecuencia levente mayor a la que aparece en el total de documentos de Brasil en LIS. Otros términos como Capabilities o Information-technology son también muy frecuentes y presentan, además, una significación superior a dos, es decir que en este conjunto aparecen el doble de veces que en el total de documentos estudiados.

La revista en la que se han publicado la mayor parte de los documentos de esta comunidad es Perspectivas em Ciências da Informação (frecuencia de $22,64 \%$ ) y una significación de 1,7 (superior a la media de la producción en LIS). Entre las revistas más habituales en esta comunidad, la que parece más ajustada a estas temáticas es Journal of Knowledge Management (significación=7,45). Las instituciones autoras de estos documentos son universidades y principalmente las de São Paulo y Minas Gerais. Se puede apreciar también que, entre las revistas más citadas aparecen principalmente títulos de management, (Tabla 5). 
Daniela De Filippo

Tabla 5 - Comunidad bibliográfica más representativa en la producción brasileña en LIS.

Table 1: The community "INNOVATION" contains $N=123$ articles. Its average internal link weight is $<\omega_{\text {in }}>\simeq 1 / 159$

\begin{tabular}{|c|c|c|c|c|c|c|c|c|}
\hline & & & Institution & $f(\%)$ & & & & \\
\hline $\begin{array}{l}\text { Reyword } \\
\text { INNOVATION }\end{array}$ & $\frac{f(\%)}{6.50}$ & $\sigma$ & UNIV SAO PAOLO & 19.51 & 1.77 & Reference & $f(\%)$ & $\sigma$ \\
\hline & 6.50 & 1.02 & UNIV FED MINAS GERAIS & & 0.65 & Nonaka I, 1997, CRIACAO CONHECIMENTO (0), 0 & 20.33 & 11.47 \\
\hline $\begin{array}{l}\text { CAPABALITTIES } \\
\text { INFORMATION-TECHNOLOGY }\end{array}$ & 5.69 & $\begin{array}{l}2.14 \\
2 \leqslant 0\end{array}$ & BELO HORTZONTE & 17.89 & 1.29 & Davenport $t \mathrm{H}, 1998$, CONHECIMENTO EMPRESA & 15.45 & 9.63 \\
\hline & $\begin{array}{l}5.69 \\
5.69\end{array}$ & 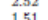 & UNIV BRASILIA & & & Nonaka I, 1995, KNOWLEDGE CREATIING C (0), 0 & 9.76 & 7.89 \\
\hline $\begin{array}{l}\text { MAANGEMENT } \\
\text { PERFORMANCE }\end{array}$ & $\begin{array}{l}5.89 \\
4.88\end{array}$ & $\begin{array}{l}1.51 \\
0.76\end{array}$ & UNIV FED SANTA CATARINA & 8.13 & 0.10 & $\begin{array}{l}\text { Choo c W, 2003, ORG CONHECIMENTO COM (0), 0 } \\
\text { Choo dun WEL } 1998 \text { KNOWING ORG ORG USE (0), }\end{array}$ & 9.76 & 5.45 \\
\hline MODEL & 4.07 & -0.72 & PROGRAMA POSGRAD CIENCIA & & & $\begin{array}{l}\text { Choo hun WET, ,998, KNOWING ORG, ORG USE (0), } \\
\text { Stewart T, 1998, CAPITAL INTELECTUAL (0), }\end{array}$ & 8.13 & $\begin{array}{l}7.12 \\
7.19\end{array}$ \\
\hline PRODUCT DEVELOPMENT & 3.25 & & & & & Nonaka I, 1994, ORGAN SCI (5), 14 CAL (0), & 8.13 & $\begin{array}{l}7.19 \\
7.19\end{array}$ \\
\hline FIRMS & 3.25 & & BR-70910900 BRASILIA & 7.32 & 0.86 & Mcgee J, 1994, GERENCIA MENTO ESTRAT (8), 0 & 8.13 & 6.75 \\
\hline TECHNOLOGY & 3.25 & -0.70 & DEPT PROD ENGN & 7.32 & 5.79 & Eisenhardt KM, 1989, ACAD MANAGE REV (14), 532 & 6.50 & 5.93 \\
\hline USER ACCEPTANCE & 3.25 & 1.90 & $\begin{array}{l}\text { DEPT CIENCIA INFORMACAO } \\
\text { UNIY FED PARAIBA }\end{array}$ & 6.50 & -0.77 & Nonaka I, 1998, CALIF MANAGE REV (40), 40 & 6.50 & 6.42 \\
\hline PERSPECTIVE & 3.25 & -0.01 & $\begin{array}{l}\text { UNIV FED PARAIBA } \\
\text { BR-88040900 FLORIANOPOLIS }\end{array}$ & 5.69 & 0.42 & Cohen WM, 1990, ADMIN SCI QUART (35), 128 & 6.50 & 5.93 \\
\hline RESOURCE-BASED VIEW & 3.25 & 1.90 & $\begin{array}{l}\text { BLORIANOPOLIS } \\
\text { FLANOPOLIS }\end{array}$ & $\begin{array}{l}5.89 \\
4.88\end{array}$ & $\begin{array}{l}0.42 \\
1.13\end{array}$ & Yin $\mathrm{r} K, 1$ 1994, CASE STUDY RES DESIG (2nd), 0 & 5.69 & 6.01 \\
\hline FIRM & 3.25 & 1.90 & BR-58059900 JOAO PESSOA & $\begin{array}{l}4.060 \\
4.07\end{array}$ & $\begin{array}{l}1.190 \\
.0 .90\end{array}$ & Hair JF, 2005, ANALISE MULTNVARIADA (0), 0 & 5.69 & 5.05 \\
\hline DYNAMIC CAPABILITIES & 3.25 & 1.90 & PORTO ALEGRE & 4.07 & 0.69 & Kogut B, 1992, ORGAN SCI (3), 383 & 4.88 & 5.56 \\
\hline $\begin{array}{l}\text { BUSINESS PERFFORMANCE } \\
\text { INFORMATION }\end{array}$ & ${ }_{2.44}^{2.44}$ & $\begin{array}{l}1.64 \\
-1.62\end{array}$ & ESCOLA CIENCIA INFORMACAO & 4.07 & -0.94 & $\begin{array}{l}\text { Yin } \mathrm{K}, 2005 \text {, ESTUDO CASO PLANEJAM (0), } 0 \\
\text { Davis FD, } 198 \text {, MIS QUART (13), 319 }\end{array}$ & 4.88 & 5.00 \\
\hline $\begin{array}{l}\text { INFORMATION } \\
\text { CREATION }\end{array}$ & 2.44 & $\begin{array}{l}-1.62 \\
1.11\end{array}$ & GETULIO VARGAS FDN & 4.07 & 5.24 & $\begin{array}{l}\text { Davis FD, } 1989, \text { MIS QUART (13), } 119 \\
\text { Alwi M 2001 MS OUART (25), } 197\end{array}$ & $\begin{array}{l}4.88 \\
4.88\end{array}$ & 5.00 \\
\hline $\begin{array}{l}\text { CREATIUN } \\
\text { STRATEGY }\end{array}$ & 2.44 & $\begin{array}{l}1.164 \\
1.64\end{array}$ & UNIV ESTADO RIO DE JANEIRO & 4.07 & 2.64 & $\begin{array}{l}\text { Alawi M, 2001, MIS QUART (25), } 107 \\
\text { Aguilar f J 1967, SCANNINC BUINESS EN ( } 0), 0\end{array}$ & $\begin{array}{l}4.88 \\
4.07\end{array}$ & 5.00 \\
\hline $\begin{array}{l}\text { IMPACT } \\
\text { IMPT }-19\end{array}$ & 2.44 & $\begin{array}{c}1.04 \\
-1.39\end{array}$ & PROGRAMA POSGRAD ENGN PROD & 3.25 & 3.53 & Grant RM, 1996, STRATEGIC MANAGE J (17), 109 & 4.07 & 5.07 \\
\hline FIRM PERFORMANCE & $\begin{array}{l}2.63 \\
1.63\end{array}$ & $\begin{array}{r}-1.39 \\
1.34\end{array}$ & $\begin{array}{l}\text { CAMPINAS } \\
\text { UNY ESTADUAL PAUUISTA }\end{array}$ & 3.25 & 0.09 & Terra j C C, 2001, GESTAO CONHECIMENTO (2), 0 & 4.07 & $\begin{array}{l}5.07 \\
5.07\end{array}$ \\
\hline Subject & $f(\%)$ & & UNIV ESIADUAL PAULASTA & 2.25 & $\begin{array}{l}-0.59 \\
\end{array}$ & & & \\
\hline Information Science \&\& Library Science & & & Country & f(\%) & $\sigma$ & CRIACAO CONHECIMENTO & & $\frac{0}{11.89}$ \\
\hline & ${ }_{17.07}$ & 9.18 & Brazil & 1000.00 & 0.37 & MIS QUART & 19.51 & 8.29 \\
\hline Computer Science, Information Systems & 3.25 & -2.62 & Una & 5.09 & 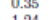 & CONḦECIMENTO EMPRESA & 19.51 & 10.66 \\
\hline Social Sciences, Interdisciplinary & 3.25 & 1.69 & Cannada & $\begin{array}{l}3.25 \\
163\end{array}$ & $\begin{array}{l}1.24 \\
.003\end{array}$ & ORGAN SCI & 19.51 & 9.89 \\
\hline Computer Science, Interdisciplinary Applications & 1.63 & -3.03 & $\begin{array}{l}\text { England } \\
\text { Portugal }\end{array}$ & $\begin{array}{l}1.63 \\
1.63\end{array}$ & 1.31 & MANAGE SCI & 18.70 & 8.02 \\
\hline & & & U arab emirate & 0.81 & 234 & ADMIN SCI QUAFT & 17.89 & 9.36 \\
\hline & & & Netherlands & 0.81 & 0.20 & ACAD MANAGE REV & 17.07 & 9.08 \\
\hline & & & South africa & 0.81 & 1.39 & STRATEGIC MANAGE J & 17.07 & 9.59 \\
\hline & & & France & 0.81 & -0.50 & $\begin{array}{l}\text { GETAOO CONHECMENTO } \\
\text { CIENCIA INFORM }\end{array}$ & $\begin{array}{l}17.07 \\
17.07\end{array}$ & 8.84 \\
\hline Journal & & & Greece & 0.81 & 1.39 & 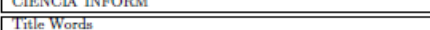 & $\begin{array}{llll}17.0 t \\
f(2)\end{array}$ & \\
\hline PERSPECT CIENC & $\frac{17 \%(1)}{27,61}$ & 1.71 & Author & I(\%) & $\sigma_{\sigma}$ & Title Words & $\frac{f(\%)}{41,16}$ & $\frac{\sigma}{0.51}$ \\
\hline TRANSINFORMACAO & 15.45 & 0.53 & Joia LA & 4.88 & 5.76 & $\begin{array}{l}\text { TNFORMAIIUN } \\
\text { KNOWLEDGE }\end{array}$ & $\begin{array}{l}41.46 \\
38.21\end{array}$ & $\begin{array}{l}0.51 \\
8.42\end{array}$ \\
\hline INFOR & 15.45 & -1.56 & Nagano & 4.88 & 5.76 & NAGEMENT & 34.15 & $\begin{array}{l}8.42 \\
11.45\end{array}$ \\
\hline & 8.13 & 7.45 & Reinharce & 4.07 & 5.26 & & 12.20 & 0.83 \\
\hline SPRACT & 4.88 & 5.76 & $\mathrm{RR}$ & 325 & 3.14 & MODEL & 11.38 & $\begin{array}{l}0.97 \\
3.97\end{array}$ \\
\hline INF SOC-ESTI & 4.88 & -0.65 & 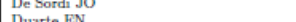 & $\begin{array}{l}2.24 \\
244\end{array}$ & 20.82 & ALYSIS & 10. & 1.57 \\
\hline INT J INFORM MANAGE & 4.07 & 3.77 & $\begin{array}{l}\text { Uluarte EN } \\
\text { Yick TE }\end{array}$ & 244 & 年 & CASE & 9.76 & 2.70 \\
\hline GOV INFORM Q & 2.44 & 3.34 & $\begin{array}{l}\text { Vick TE } \\
\text { Lounad M }\end{array}$ & 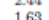 & 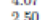 & & 8.94 & -0.13 \\
\hline SOC SCI INFORM & 2.44 & 1.24 & Muniz J & $\begin{array}{lll}1.63 \\
1.63\end{array}$ & 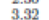 & ORGANIZATIONAL & 8.94 & 6.65 \\
\hline & 2.44 & 2.82 & Do Amaral SA & 1.63 & 250 & & 8.13 & \\
\hline
\end{tabular}

Fuente: extracción directa de Bibliotools

La comunidad dos está compuesta por 111 artículos y muestra un marcado contenido cienciométrico. A través de las palabras clave se observa que destacan términos como: impact, indicators y H-index. La revista Scientometrics es el principal medio de difusión y tiene una importancia central con una alta frecuencia $(\mathrm{f}=49,55 \%)$ y una elevada significación $(17,81)$. Es también la revista más utilizada en las referencias $(\mathrm{f}=71,17 \%)$. Las principales instituciones autoras son las universidades USP, UFRJ y UNICAM. Entre los autores más citados se encuentran claros referentes internacionales del ámbito de la cienciometría: Glanzel, Hirsch, Katz y destacados autores brasileños que trabajan en este campo: Leta, Meneghini, etc. (Tabla 6).

La comunidad bibliográfica número tres se vincula con temáticas sobre organización del conocimiento y clasificación, como se puede apreciar por las principales palabras clave que la definen. Las revistas de publicación más relevantes son brasileñas: Transinformação, Perspectivas em Ciências da Informação e Informação \& Sociedade: Estudos, pero se utilizan como referente, en general, revistas extranjeras: Journal of Documentation, Journals of the America Society for Information Science (Tabla 7). 
Daniela De Filippo

Tabla 6 - Comunidad bibliográfica número dos en la producción brasileña en LIS.

Table 2: The community "SCIENCE" contains $N=111$ articles. Its average internal link weight is $<\omega_{\text {in }}>\simeq 1 / 147$

\begin{tabular}{|c|c|c|c|c|c|c|c|c|}
\hline & & & & & & & & \\
\hline $\begin{array}{l}\text { Reyword } \\
\text { SCIENCE }\end{array}$ & $\frac{f(\%)}{24.32}$ & $\frac{\sigma}{209}$ & UNIV SAO PAOLO & $\frac{10 \% 1}{23.42}$ & 2.87 & \begin{tabular}{|l} 
Recterence \\
Glanzel W, 2006, SCIENTOMETRICS \\
\end{tabular} & $\frac{H(7)}{1441}$ & \\
\hline JOURNALS & 10.81 & 3.49 & UNIV FED RIO DE JANEIRO & 14.41 & 2.62 & Hiresh JE, 2005, P NATL ACAD SCI USA (102), 16569 & 11.71 & \\
\hline IMPACT & 9.01 & 1.79 & UNIV ESTADUAL CAMPINAS & 14.41 & 5.60 & Leta J, 2006, SCIENTOMETRICS (67), 87 ( & 10.81 & 8.44 \\
\hline INDICATORS & 8.11 & 2.87 & BELO HORZONTE & 7.21 & -2.03 & Katz JS, 1997, RES POLICY (26), 1 & 9.01 & 7.23 \\
\hline COLLABORATION & 6.31 & 2.83 & ONIV FED RIO GRANDEE DO SUL & 7.21 & 20.88 & Spinak E, 1998, CIENCIA INFORM BRASI (27), 141 & 8.11 & \\
\hline PUBLICATIONS & 5.41 & 2.62 & $\begin{array}{l}\text { UNIV FED MINAS GERAIS } \\
\text { UNIV BRASIIA }\end{array}$ & $\begin{array}{l}6.31 \\
5.41\end{array}$ & -2.73 & Leta J, 2001, SCIENTOMETRICS (50), 241 & 6.31 & \\
\hline PATTERNS & 5.41 & 1.54 & UNIV FED SAO CARLOS & 5.41 & 2.72 & Meneghini R, 1996, SCIENTOMETRICS (35), 367 & 6.31 & \\
\hline QUALITY & 4.50 & 1.56 & CAMPINAS & 4.50 & 0.85 & Leta J, 1996, SCIENTOMETRICS (35), 33 & 6.31 & 6.43 \\
\hline $\begin{array}{l}\text { INNOVATION } \\
\text { INDEX }\end{array}$ & $\begin{array}{l}4.50 \\
3.50\end{array}$ & -0.04 & BR-70910900 BRASILIA & 4.50 & -0.48 & $\begin{array}{l}\text { Narin F, 1997, RFS POLICY (26), 317 } \\
\text { W POL }\end{array}$ & 5.41 & 5.95 \\
\hline $\begin{array}{l}\text { INDEX } \\
\text { CITATION }\end{array}$ & $\begin{array}{l}3.60 \\
3.60\end{array}$ & $\begin{array}{r}2.13 \\
2.13\end{array}$ & INST PHYS SAO CARLOS & 4.50 & 5.60 & 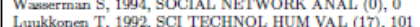 & 5.41 & 5.37 \\
\hline 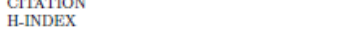 & $\begin{array}{l}3.60 \\
3.60\end{array}$ & $\begin{array}{l}2.13 \\
2.13\end{array}$ & PROGRAMA POSGRAD CIENCIA & & & 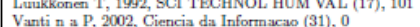 & 4.50 & $\begin{array}{l}5.42 \\
4.32\end{array}$ \\
\hline TECHNOLOGY & 3.60 & $\begin{array}{l}2.199 \\
-0.49\end{array}$ & $\begin{array}{l}\text { INFORMACAO } \\
\text { INPMCAO }\end{array}$ & 4.50 & -3.16 & Leta J, 2002, SCIENTOMETRICS (53), 325 , & 4.50 & 5.42 \\
\hline RANKING & 3.60 & 2.13 & DEPT CIENCLA INFORMACAO & 4.50 & -1.49 & Newman MEJ, 2001, PHYS REV E (64), 0 & 4.50 & 5.42 \\
\hline COMPLEX NETWORKS & & & FUNDACAO OSWALDO CRUZ & 3.600 & 2.09 & Figueira I, 2003, SCIENTOMETRICS (56), 317 & & 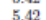 \\
\hline INTERNATIONAL COLLABORATION & 3.60 & 2.13 & INST CIENCIAS BIOMED & 3.60 & 5.01 & Let3 J, 1998 , SCIENTOMETRICS (41), 313 & 4.50 & 5.42 \\
\hline KNOWLEDGE & 2.70 & -0.33 & DEPT BIOQUIM MED & 3.60 & 5.01 & Okubo $\mathrm{Y}, 1997$, BIBLIOMETRIC INDICAT (0), 0 & 3.60 & 4.85 \\
\hline SCIENTIFIC COOPERATION & 2.70 & 1.84 & CTR CIENCIAS SAUDE & 3.60 & 3.81 & Costa LD, 2007 , ADV PHYS (56), 167 & 3.60 & 4.85 \\
\hline IMPACT FACTOR & 2.70 & 1.84 & $\begin{array}{l}\text { NSST BIOQQUM MED } \\
\text { UNIY FED SAO PAULO }\end{array}$ & $\begin{array}{l}3.60 \\
3.60\end{array}$ & $\begin{array}{l}3.39 \\
276\end{array}$ & Gibbs W W, 1995 , SCI AM $(273), 76$ & 3.60 & 4.85 \\
\hline EVOLUTION & 2.70 & 1.29 & $\begin{array}{l}\text { UNIV FED SAO PAULO } \\
\text { BR-13083852 CAMPINAS }\end{array}$ & $\begin{array}{l}3.60 \\
3.60\end{array}-10$ & $\begin{array}{l}2.766 \\
4.32\end{array}$ & Albert R, 2002, REV MOD PHYS (74), 47 & 3.60 & 4.85 \\
\hline Subject & $f(\%)$ & & 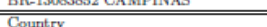 & $\begin{array}{ll}3.00 \\
f(\%)\end{array}$ & 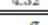 & "RefJournal & $\overline{f(\%)}$ & \\
\hline Information Science \& Library Science & 100.00 & 0.00 & Country & $\frac{f(\%)}{10000}$ & $\frac{\sigma}{0.25}$ & SCIENTOMETRICS & 71.17 & 20.03 \\
\hline Computer Science, Interdisciplinary A pplications & 51.35 & 14.80 & $\begin{array}{l}\text { Brazil } \\
\text { Unas }\end{array}$ & $\begin{array}{r}100.00 \\
9.01\end{array}$ & $\begin{array}{l}0.85 \\
1.94\end{array}$ & NATURE & 29.73 & \\
\hline Computer Science, Information Systems & 9.01 & -0.51 & Esa & $\begin{array}{l}9.01 \\
5.41\end{array}$ & $\begin{array}{l}1.94 \\
308\end{array}$ & RES POLICY & 27.03 & 10.13 \\
\hline Medical Informatics & 1.80 & -0.56 & Spain & 400 & 024 & SCIENCE & 25.23 & 9.92 \\
\hline Management & 0.90 & -1.29 & Canada & 1.80 & 0.02 & J AM SOC INF SCI TEC & 24.32 & 4.62 \\
\hline 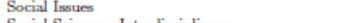 & 0.90 & 2.51 & Switzerland & 1.80 & 3.54 & P NATL ACAD SCI USA & 20.72 & 10.86 \\
\hline Social Sciences, Interdisciplinary & 0.90 & -0.47 & $\begin{array}{l}\text { Belgium } \\
\text { nom }\end{array}$ & 1.80 & 1.47 & J INFORMETR & 14.41 & 9.77 \\
\hline & & & Hungary & 1.80 & 2.15 & $\begin{array}{l}\text { BRAZ J MED BIOL RES } \\
\text { CIENCIA INFORM }\end{array}$ & ${ }_{1351}^{14.41}$ & 9.77 \\
\hline & & & South korea & 1.80 & 2.69 & $\begin{array}{l}\text { CIENCIA INFOTM } \\
\text { RES EVALUAT }\end{array}$ & $\begin{array}{l}13.51 \\
13.51\end{array}$ & $\begin{array}{l}-1.64 \\
9.07\end{array}$ \\
\hline Journal & $f(\%)$ & & Netherlands & 0.90 & 0.30 & Title Words & $=18(\%)$ & \\
\hline SCIENTOMETRICS & 49.55 & 17.81 & Author & $f(\%)$ & $\sigma$ & BRAZILIAN & 27.93 & $\frac{\sigma}{6.77}$ \\
\hline PERSPECT CIENC INF & 11.71 & -2.47 & $\begin{array}{l}\text { Leta J } \\
\text { Wainer J }\end{array}$ & $\begin{array}{l}9.01 \\
5.41\end{array}$ & 6.37 & SCIENTIFIC & 26.13 & 6.42 \\
\hline INFORM SOC-ESTUD & 6.31 & -3.84 & $\begin{array}{l}\text { Wanter } \mathrm{J} \\
\text { Costa LD }\end{array}$ & $\begin{array}{l}5.41 \\
4.50\end{array}-10$ & $\begin{array}{l}6.16 \\
5.62\end{array}$ & INFORMATION & 18.02 & -4.58 \\
\hline J INFORMETR & 5.41 & 5.09 & Mugnaini $\mathrm{R}$ & 3.60 & 4.34 & STUDY & 16.22 & 2.21 \\
\hline TRANSINFORMACAO & 4.50 & -2.84 & Figueira I & 3.60 & 5.02 & SCIENCE & 16.22 & 1.39 \\
\hline $\begin{array}{l}\text { RES EVALUAT } \\
\text { INF SOC-DSTUD }\end{array}$ & 2.70 & 3.06 & Amancio DR & 2.70 & 4.25 & 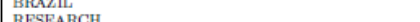 & ${ }_{16.24}^{16.22}$ & 3.15 \\
\hline $\begin{array}{l}\text { INF SOC-ESTUD } \\
\text { INF DEV }\end{array}$ & $\begin{array}{l}2.70 \\
1.80\end{array}$ & $\begin{array}{r}-1.56 \\
2.69\end{array}$ & De Souza CG & 2.70 & 4.35 & $\begin{array}{l}\text { RESEARCH } \\
\text { ANALYSIS }\end{array}$ & $\begin{array}{l}14.41 \\
14.41\end{array}$ & $\begin{array}{l}3.84 \\
3.09\end{array}$ \\
\hline $\mathrm{J}$ AM MED INFORM ASSN & 1.80 & -0.56 & Bini LM & 2.70 & 4.35 & $\begin{array}{l}\text { ANATWEEN } \\
\text { BETWE }\end{array}$ & 13.51 & 4.34 \\
\hline ASLIB PROC & 1.80 & 3.55 & $\begin{array}{l}\text { Pinto AL } \\
\text { Oliveir ON }\end{array}$ & $\begin{array}{l}2.70 \\
2.70\end{array}$ & 2.655 & PRODUCTION & 9.01 & 2.91 \\
\hline
\end{tabular}

Fuente: extracción directa de Bibliotools

Tabla 7 - Comunidad bibliográfica número tres en la producción española en LIS.

Table 3: The community "INFORMATION-SCIENCE" contains $N=103$ articles. Its average internal link weight is $<\omega_{\text {in }}>\simeq 1 / 274$

\begin{tabular}{|c|c|c|c|c|c|c|c|c|}
\hline & & & & & & \begin{tabular}{|l|l|l|l|l} 
Reference & \\
\end{tabular} & $\mathrm{f}(\%)$ & \\
\hline & & & Institution & $f(\%)$ & $\sigma$ & Hjorland B, 2002, JDOC (58), 422 & 9.71 & \\
\hline $\begin{array}{l}\text { Keyword } \\
\text { INFORMATION-SCIENCE }\end{array}$ & 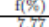 & & UNIV FED MINAS GERAIS & 23.30 & 2.10 & 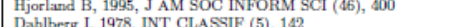 & 8.74 & 6.00 \\
\hline & ${ }_{5.83}^{7.77}$ & $\begin{array}{l}2.247 \\
2.47\end{array}$ & PROGRAMA POSGRAD CIENCIA & & & 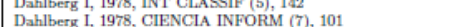 & 6.80 & $\begin{array}{l}7.21 \\
6.19 \\
-10\end{array}$ \\
\hline CLASSIFICATION & 3.88 & -2.30 & $\begin{array}{l}\text { INFORMACAO } \\
\text { BELO HORZONTE }\end{array}$ & $\begin{array}{l}21.36 \\
11.45\end{array}$ & 1.70 & $\begin{array}{l}\text { Cintra anna maria MARQUES, 2002, ENTENDER } \\
\text { ENTER }\end{array}$ & & \\
\hline KNOWLEDGE ORGANIZATION & 2.91 & 1.99 & BELO HORZONTE & 18.45 & 1.34 & LINGUAGENS $(0), 0$ & & \\
\hline INFORMATION & 2.91 & -1.28 & $\begin{array}{l}\text { DEPT CIENCIA INFORMACAO } \\
\text { UNY FED SANTA CATARINA }\end{array}$ & $\begin{array}{l}18.45 \\
18.25\end{array}$ & 3.66 & Lanciaster F, 1986, VOCABULARY CONTROL I (0), 0 & ${ }_{5.83}^{6.00}$ & 6.24 \\
\hline DOMAIN-ANALYSIS & 2.91 & 1.99 & $\begin{array}{l}\text { UNIV FED SANTA CATARINA } \\
\text { ESCOLA CIENCIA INFORMACAO }\end{array}$ & 13.59 & 2.15 & Guarino $N$, 1995, VERY LARGE KNOWLEDGE (0), 25 & 5.83 & 6.24 \\
\hline SYSTEMS & 2.91 & .0 .81 & $\begin{array}{l}\text { ESCLLA CIINCLA INFORMACAO } \\
\text { UNIV SAO PAULO }\end{array}$ & 10.68 & 1.94 & Gruber TR, 1993, KNOWL ACQUIS (5), 199 & 5.83 & 6.24 \\
\hline INFORMATION-RETRIEVAL & 1.94 & 0.57 & $\begin{array}{l}\text { UNIV SAO PAULO } \\
\text { UNIV ESTADUAL PAULISTA }\end{array}$ & $\begin{array}{l}10.68 \\
971\end{array}$ & -0.978 & Guarino N, 1998, FORMAL ONTOLOGY INFO $(0), 0$ & 5.83 & 6.24 \\
\hline THESAURI & 1.94 & 1.62 & $\begin{array}{l}\text { UNIV ESTADUALL PAULLISTA } \\
\text { BR-88040900 FLOPIANOPOLIS }\end{array}$ & 9.71 & 2.68 & Hjorland B, 2003, KNOWL ORGAN (30), 87 & 4.85 & 5.69 \\
\hline RETRIEVAL & 1.94 & -1.28 & $\begin{array}{l}\text { BR-88000900 FLORLANOPOLIS } \\
\text { BR-31270901 BELO HORIZONTE }\end{array}$ & $\begin{array}{l}7.77 \\
5.83\end{array}$ & $\begin{array}{l}1.36 \\
2.63\end{array}$ & Houaiss A, 2001 , DICIONARIO HOUAISS L $(0), 0$ & 4.85 & 5.69 \\
\hline REPRESENTATION & 1.94 & & 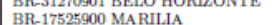 & $\begin{array}{l}5.85 \\
4.85\end{array}$ & ${ }_{4.71}^{2.63}$ & Fonseca F, 2007, J AM SOC INF SCI TEC (58), 786 & & \\
\hline ORGANZATION SYSTEMS & 1.94 & 0.99 & DEPT INFORMAT SCI & 4.85 & 2.53 & Vickery BC, 1997 , J INF SCI (23), 227 & 4.85 & 5.69 \\
\hline DOCUMENTS & 0.97 & 1.15 & UNIV FED FLUMINENSE & 4.85 & ${ }_{1.81}^{2.83}$ & Lancister f W, 1993, INDEXACAO RESUMOS TE (0), 0 & 4.85 & 5.06 \\
\hline $\begin{array}{l}\text { KNOWLEDGE } \\
\text { RESEARCH OUESTIONS }\end{array}$ & 0.97 & -1.31 & FLORIANOPOLIS & 4.85 & 1.02 & Ranganathan shyali R, 1967, PROLEGOMENA LIB CLAS & & \\
\hline $\begin{array}{l}\text { RESEARCH QUESTIONS } \\
\text { PRINCIPLES }\end{array}$ & 0.97 & 1.15 & SCH INFORMAT SCI & 4.85 & 3.63 & $\begin{array}{l}(0), 0 \\
\text { Burdung }\end{array}$ & 4.85 & 5.69 \\
\hline $\begin{array}{l}\text { PRIINCIPLES } \\
\text { CONTEXT }\end{array}$ & 0.97 & 1.15 & UNIV FED RIO DE JANEIRO & 4.85 & -1.11 & 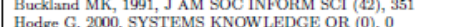 & 4.85 & 277 \\
\hline $\begin{array}{l}\text { CONTEXT } \\
\text { CATEGOES }\end{array}$ & $\begin{array}{l}0.97 \\
0.97\end{array}$ & $\begin{array}{l}1.15 \\
1.15\end{array}$ & FAC FILOSOFIA \& CIENCLAS & 4.85 & 3.63 & 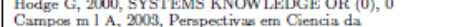 & & \\
\hline CORPORATE MEMORY & 0.97 & 1.15 & DEPT COMP SCI & 4.85 & 0.64 & 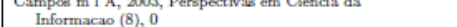 & 3.88 & 5.09 \\
\hline CATEGORZZATION & 0.97 & 1.15 & UNIV FED SAO CARLOS & 3.88 & 1.49 & 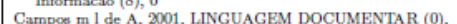 & 3.088 & \\
\hline Subject & $=f(\%)$ & & $\begin{array}{l}\text { SAO PAULO STATE UNIV UNESP } \\
\text { C }\end{array}$ & 3.88 & 5.25 & 0 & 3.88 & \\
\hline Information Science \& Library Science & 100.00 & & Country & $f(8)$ & & Vickery BC, 1960, FACETED CLASSIFICATI (0), 0 & 3.88 & 5.09 \\
\hline Computer Science, Information Systems & 7.77 & -0.90 & Brazil & 100.00 & 0.34 & RefJournal & If(\%) & \\
\hline Education \& Educational Research & 0.97 & 1.62 & Spain & 10.68 & 2.65 & J DOC & & \\
\hline & & & $\mathrm{Us}_{\mathrm{s}}$ & 3.88 & -0.52 & J AM SOC INFORM SCI & 25.24 & 3.67 \\
\hline & & & minada & 2.91 & 0.87 & IA INFORM & 21.36 & 0.42 \\
\hline & & & France & 1.94 & 0.54 & VL ORG & 20.39 & 1054 \\
\hline & & & England & 0.977 & -0.55 & J AM SOC INF SCI TEC & 16.50 & 1.89 \\
\hline & & & Uruguay & 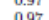 & 2.62 & INFORM PROCESS MANAG & 12.62 & 0.92 \\
\hline & & & Germentany & 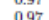 & 0.57 & TRANSINFORMACAO & 12.62 & 2.30 \\
\hline & & & Wales & 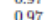 & 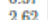 & Ciencia da Infornacao & 12.62 & -0.44 \\
\hline Journal & 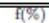 & & wales & 0.97 & 2.62 & LIBR TRENDS & 11.65 & 4.37 \\
\hline TRANSINFORMACI & & & Author & $\frac{f(\%)}{680}$ & $\sigma$ & INT CLASSIF & 10.68 & 8.47 \\
\hline PERSPECT CIEN & 22.33 & 0.25 & Fujita MS & 6.80 & & Title Words & $f(\%)$ & $\bar{\sigma}$ \\
\hline INFORM SOC-ESTUD & 19.42 & -0.45 & Limai GA & 5.88 & 6.46 & INFORMATT & & -1.90 \\
\hline KNOWL ORGA & 16.50 & 8.51 & $\begin{array}{l}\text { Almeida MB } \\
\text { Orteg CD }\end{array}$ & 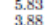 & $\begin{array}{l}4.949 \\
5.97\end{array}$ & KNOWLEDGE & 23.30 & 3.18 \\
\hline & 6.80 & 0.21 & $\mathrm{Ort}$ & 3.38 & $\begin{array}{l}5.27 \\
4.56\end{array}$ & ORGANIZATION & 13.59 & 6.51 \\
\hline INFORM PROCESS MANAG & 2.91 & 0.67 & $\begin{array}{l}\text { Cale L } \\
\text { Marcondes } \mathrm{CH}\end{array}$ & 3.88 & $\begin{array}{l}4.566 \\
4.56\end{array}$ & SYSTEMS & 11.65 & 4.33 \\
\hline PROF INFORM & 1.94 & 1.35 & Souza RR & 2.91 & 2.81 & SCIENCE & 10.68 & -0.39 \\
\hline & & & & 2.91 & 3.78 & ONTOLOGY & 8.74 & 6.23 \\
\hline $\begin{array}{l}\text { AMT SOC INF SCI TEC } \\
\text { INT J COMPSUPP COLL }\end{array}$ & $\begin{array}{l}1.94 \\
0.97\end{array}$ & $\begin{array}{l}0.05 \\
1.62\end{array}$ & & 2.91 & 4.56 & $\begin{array}{l}\text { REPRESENTATION } \\
\text { ONTOLOGIFS }\end{array}$ & 年.77 7.77 & ${ }_{746}^{401}$ \\
\hline & & & Cafe LMA & 2.91 & 3.78 & $\begin{array}{l}\text { STUDY } \\
\text { STOES }\end{array}$ & 7.77 & -0.74 \\
\hline
\end{tabular}

Fuente: extracción directa de Bibliotools

Las restantes comunidades agrupan un número inferior de documentos (ente 14 y 94) de temáticas no tan homogéneas. A pesar de que su contenido es 


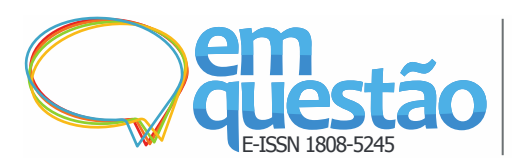

¿Qué publica Brasil en Biblioteconomía y documen-

tación? Estudio de la producción internacional y

análisis de Clúster Bibliográficos (Web of Science 2000-

2014)

Daniela De Filippo

más variado los principales tópicos en torno a los que se agrupan son: recuperación de información / estudios de usuarios (CB 5), estudios sobre web e internet (CB 6), Open Access / revistas electrónicas (CB 7), estudios de comunicación científica en Brasil (CB 8), estudios métricos de la información / ciencias de la información generalista (CB 4 y 9), metodologías en biblioteconomía y documentación (CB 10).

A través de diferentes programas de visualización es posible comprobar las relaciones entre las diferentes variables presentadas en las tablas anteriores. Así, con el programa Gephi (versión 0.8.2) se muestran los vínculos entre las disciplinas (nodos azules), las revistas de publicación (nodos violetas) y las revistas de referencia (nodos naranjas). El tamaño de los nodos es proporcional al número de documentos que concentra. Se aprecia que la disciplina de mayor presencia es Information Science and Library Science, ya que todos los documentos pertenecen a ella. Sin embargo, aparecen otras como Computer Science, Interdisciplinary Application, Management, etc. Se observa también que las tres revistas brasileñas son las más importantes como vehículos de difusión, mientras que otras como Scientometrics son las más referenciadas (Figura 4). 
Daniela De Filippo

Figura 4 - Relaciones entre disciplinas, revistas de publicación y revistas referenciadas en la producción de Brasil en LIS (WoS 2000-2014).

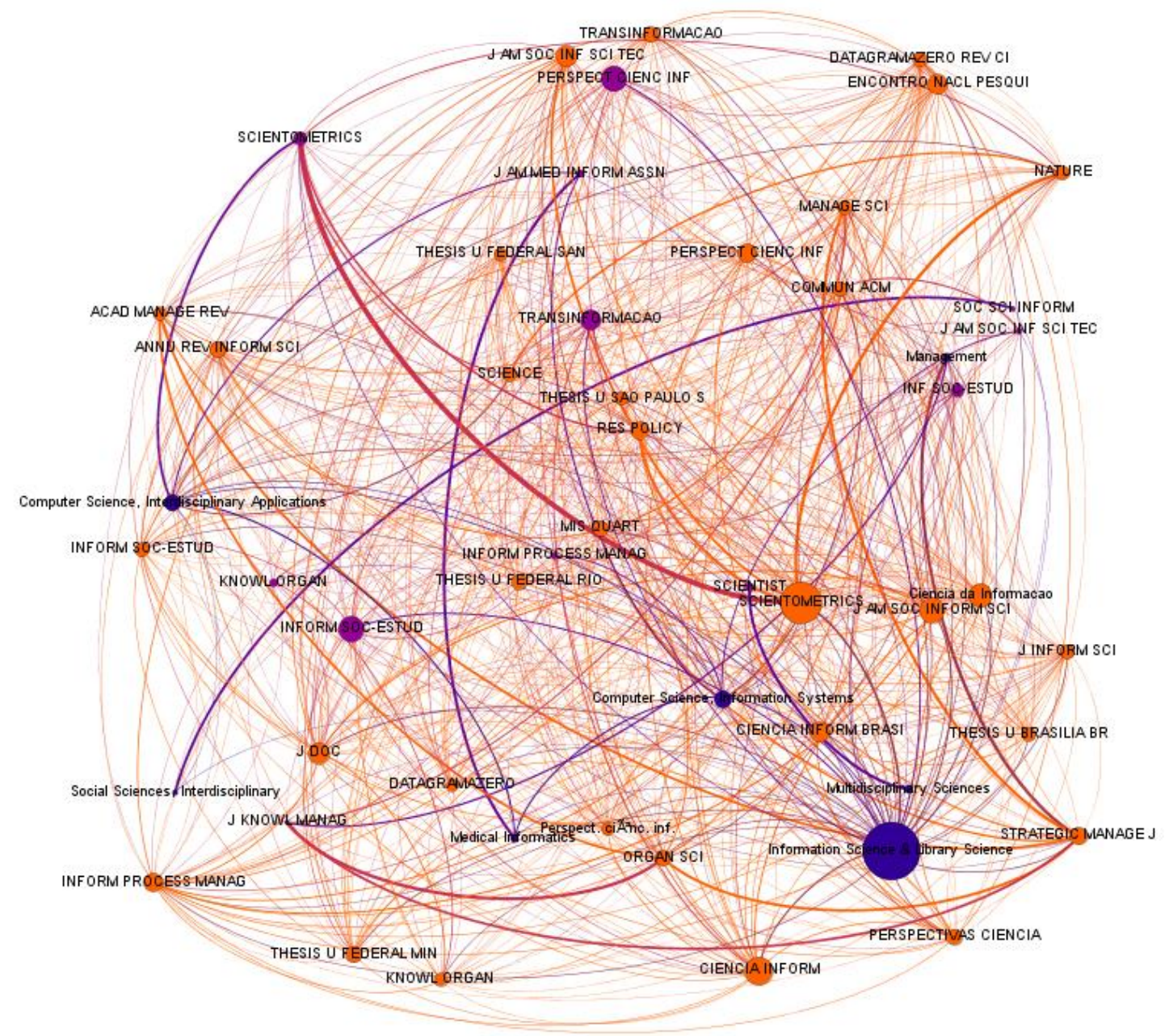

Fuente: Elaboración propia a partir de datos obtenidos de Bibliotools

En la figura 5 se muestran también las relaciones entre comunidades bibliográficas. Cada nodo representa una comunidad y el tamaño está en función del número de documentos que contiene. Las aristas muestran las relaciones entre comunidades, establecidas a partir de las referencias compartidas, y su grosor indica el volumen de referencias comunes. Así, se observa que existen fuertes vínculos entre las comunidades 4-5 y 4-6, dado que la 4 toca temas gene- 
Daniela De Filippo

rales. También son frecuentes los vínculos entre las comunidades uno y cinco (Figura 5).

Figura 5 - Comunidades bibliográficas en la producción de Brasil en LIS (WoS 2000-2014).

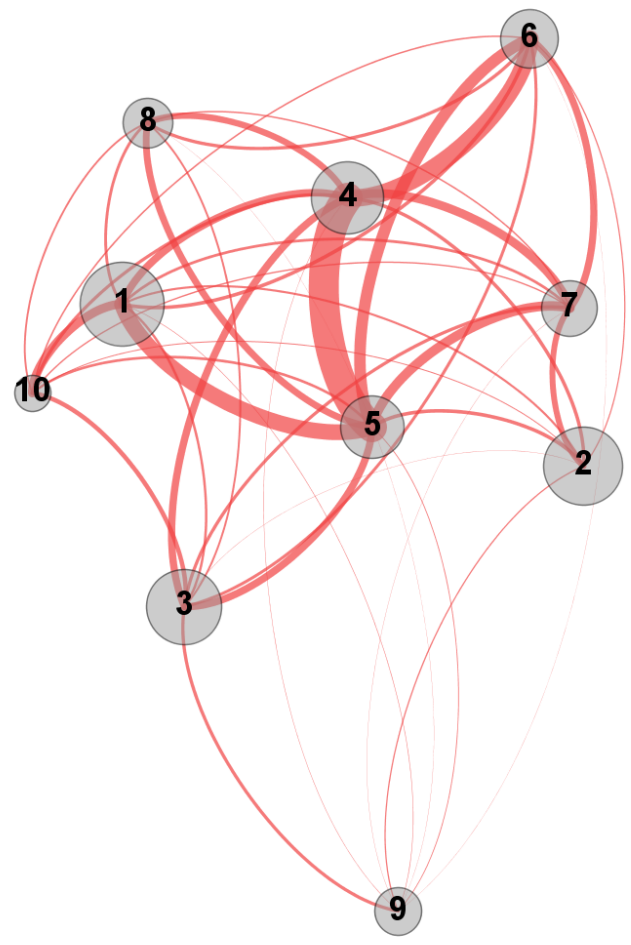

Fuente: Elaboración propia a partir de datos obtenidos de Bibliotools

\section{Comentarios finales}

El estudio de la producción de Brasil en Library \& Information Science muestra que, a pesar de contar con una escasa aportación en términos absolutos, la posición del país en esta disciplina es mejor que la del total de la producción frente al mundo. Aunque la producción en este campo está muy concentrada en el ámbito anglosajón, las publicaciones Iberoamericanas van cobrando espacio. En el caso de Brasil la difusión de los resultados en revistas del mainstream science se presenta como una tendencia reciente que coincide con el ingreso a la web of science de dos revistas nacionales del campo (Informação \& Sociedade: Estudos y Transiformação) y otras de diversas disciplinas que también recogen producción de investigadores del área. 


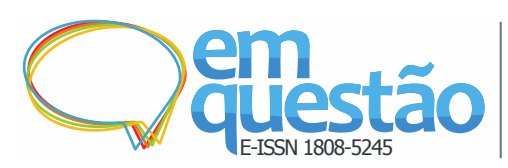

¿Qué publica Brasil en Biblioteconomía y documen-

tación? Estudio de la producción internacional y

análisis de Clúster Bibliográficos (Web of Science 2000-

2014)

Daniela De Filippo

Este incremento en la producción internacional brasileña en LIS en los últimos años puede entenderse, en parte, por el desarrollo de estudios universitarios de grado y postgrado específicos del área. Asimismo, la investigación en LIS ha tenido también un importante crecimiento gracias a la actividad de grupos de investigación - especialmente vinculados con universidades y centros estatales de I+D - con una orientación muy internacional. Esto se suma a la importancia que va adquiriendo la publicación en revistas indexadas en Web of Science como criterio de evaluación de los investigadores, lo que ha producido un crecimiento muy acelerado de las publicaciones en esta base de datos.

En cuanto a las características formales de la producción, son notables las diferencias idiomáticas en las publicaciones LIS de Brasil frente al mundo. Mientras casi un $60 \%$ de las publicaciones del país se realizan en idioma local, el portugués representa menos de un $0,5 \%$ de la producción mundial en LIS. Esto puede estar dificultando la difusión de la actividad investigadora fuera de las fronteras. De hecho, las tres principales revistas de publicación son editadas en Brasil y en ellas una amplia mayoría de los documentos provienen de instituciones nacionales. Estas revistas concentran el $60 \%$ de la producción de Brasil en LIS, mientras que sólo recogen un $0,5 \%$ de la producción de otros países.

Las publicaciones brasileñas en este campo han sido clasificadas también en otras disciplinas entre las que destaca Computer Science and Information System, al igual que en el conjunto de la producción del mundo. Sin embargo, otras disciplinas muy presentes en la producción del país, son menos representativas en el total del mundo como Computer Science, Interdisciplinary Applications y Social Science Interdisciplinary.

La segunda fase del estudio se ha centrado en el análisis de Comunidades Bibliográficas que ha permitido detectar las relaciones intertextuales y determinar los principales referentes bibliográficos. En este sentido, es interesante observar que en Brasil existen campos de investigación muy variados dentro de la producción LIS. Entre las comunidades bibliográficas que agrupan mayor número de publicaciones se advierte que la presencia de revistas brasileñas es muy alta, tanto como medio de difusión como de referencia. La comunidad bibliográfica relacionada con temas cienciométricos $\left(\mathrm{CB} \mathrm{N}^{\circ} 2\right)$ es quizá la más interna- 
cional ya que utiliza como referentes principales a autores y revistas extranjeras. Por el contrario, otras comunidades se centran en el estudio de casos locales. Aunque se trata de un conjunto documental que podría ser considerado homogéneo, cada comunidad presenta características propias. Si bien existe interacción a través de las $\mathrm{CB}$, dado que comparten referencias bibliográficas, no es posible afirmar que los autores brasileños en LIS tengan un marco teórico uniforme. Por el contrario, en cada sub-área los referentes son diversos y conviven autores clásicos, como Bourdieu o Foucault, con otros contemporáneos del país o el ámbito internacional.

Si bien es evidente que la producción científica en Library \& Information Science recogida en Web of Science es sólo una porción de lo que se publica en esta disciplina, este estudio nos permite conocer cuáles son las líneas más internacionales que se difunden a través de revistas del mainstream science. Los datos obtenidos evidencian que, a pesar de tratarse de una disciplina joven, en los últimos cinco años ha habido un incremento notable del número de documentos de Brasil. Esta tendencia parece que seguirá acentuándose debido al crecimiento científico del país en los últimos años que, indudablemente, tendrá repercusiones en el ámbito de la publicación científica.

\section{Referencias}

ANDRÉ, C. S. et al. Análise bibliométrica do periódico TransInformação. Profesional de la Información, Barcelona, v. 23, n. 4, p.433-442, jul./ago. 2014.

FORESTI, N. A. B. Contribuição das revistas brasileiras de Biblioteconomia e Ciência da Informação enquanto fonte de referência para pesquisa. Ciência Informação, Brasília, v.19, n. 1, p. 53-71, jan./jun. 1990.

GRAUWIN, S. et al. Complex systems science: dreams of universality, interdisciplinarity reality. Journal of the American Society for Information Science and Technology, Heboken, v. 63, n. 7, p.1327-1338, 2012.

GRAUWIN, S. Create your own map of sciences! c2012. Disponível em: <http://www.sebastian-grauwin.com/?page_id=427>.Acesso em: 6 out. 2015.

GRAUWIN, S.; JENSEN, P. Mapping scientific institutions. Scientometrics, Amsterdam, v. 89, n. 3, p. 943-954, dez. 2011. 
Daniela De Filippo

KESSLER, M. M. Bibliographic coupling between scientific papers. American Documentation, Washington, v. 14, n. 1, p.10-25, 1963.

LEVITT, J. M.; THELWALL, M. The most highly cited Library and Information Science articles: interdisciplinarity, first authors and citation patterns. Scientometrics, Amsterdam, v. 78, n. 1, p.45-67, jan. 2009.

NIXON, J. M. Core journals in Library and Information Science: developing a methodology for ranking LIS journals. College \& Research Libraries, Chicago, v. 75, n. 1, p. 66-90, jan. 2014. Disponível em:

$<$ http://docs.lib.purdue.edu/cgi/viewcontent.cgi?article $=1075 \&$ context=lib_fsdo cs>. Acesso em: 6 out. 2015.

OLIVEIRA, E. F. T.; MORAES, J. B. E. Evaluación de la producción científica de las revistas de ciencia de la información sobre el tema estudios métricos en SciELO. Ibersid: revista de sistemas de información y documentación, Zaragoza, v. 2, p.109-115, 2008. Disponível em: <http://ibersid.eu/ojs/index.php/ibersid/article/view/2213/1974>. Acesso em: 6 out. 2015.

PINTO, M. D. S.; SANTOS, R. N. M.; BAHIA, E. M. Análise de citação da revista eletrônica Arquivística.net: uma aplicação das técnicas bibliométricas. Em Questão, Porto Alegre, v. 15, n. 1, p. 27-42, jan./jun. 2009.

SOUZA, C. G.; FERREIRA, M. L. A. Researchers profile, co-authorship pattern and knowledge organization in Information Science in Brazil. Scientometrics, Amsterdam, v. 95, n. 2, p. 673-687, 2013.

THOMSOM REUTERS. Essential science indicators. 2015a.

THOMSOM REUTERS. Journal Citation Report. 2015 b.

\title{
What is publishing Brazil in Library and Information Science? Study of international papers and clustering analysis (Web of Science 2000 to 2014)
}

\begin{abstract}
This article analyses publications from Brazil in Library and Information Science (Web of Science). The main objectives are: to know the production's characteristics, to analyze the country's profile of activity and to detect the documentary corpus used as a conceptual framework. The methodology consists of two stages. At first, papers were collected and the main bibliometric indicators of activity, specialization and collaboration were obtained. Bibliographic coupling method was used in the second stage to detect "Bibliographical communities" with common references. The results show that 910 documents have been published by Brazilian centers between 2000 and 2014, especially in
\end{abstract}


Daniela De Filippo

Portuguese, wherein the main dissemination ways are national magazines. Different bibliographic clusters were detected with specific topics, authors and related reference: Open Access studies, information retrieval, metric studies, knowledge organization and scientometrics. The latter community is the most international, due to both consumption and diffusion magazines.

Keywords: Scientific production. Bibliographic clusters. Information and Library Science. Brazil

\section{O que o Brasil publica em Biblioteconomia e Documentação?}

Estudo da produção internacional e análise de clusters bibliográ-

\section{ficos (Web of Science 2000-2014)}

Resumo: Neste trabalho analisam-se as publicações do Brasil em Library and Information Science da Web of Science. O objetivo é conhecer as características da produção, analisar o perfil de atividade do país e detectar o corpus documental utilizado como arcabouço conceitual. A metodologia consiste em duas etapas: na primeira coletou-se a produção e obtiveram-se os principais indicadores bibliométricos de atividade, especialização e colaboração. Na segunda, utilizouse o método de bibliographic coupling para detectar "comunidades bibliográficas" com referências comuns. Os resultados mostram que os centros brasileiros publicaram 910 documentos entre 2000 e 2014, especialmente em português, sendo que as revistas editadas no país são os principais canais de difusão. Detectaram-se clusters bibliográficos diferenciados, com temáticas e autores de referência específicos e relacionados com estudos sobre Open Access, recuperação da informação, estudos métricos, organização do conhecimento e cienciometria. Essa última comunidade é a mais internacional, tanto pelas revistas de consumo quanto de difusão.

Palavras-chave: Produção científica. Clústers bibliográficos. Informação e Documentação. Brasil.

Recebido em: 09/09/2015

Aceito em: 17/12/2015 